\title{
DISFUNCIÓN DIAFRAGMÁTICA ASOCIADA A LA VENTILACIÓN MECÁNICA INVASIVA EN PACIENTES ADULTOS CRÍTICAMENTE ENFERMOS
}

\author{
DIAPHRAGMATIC DYSFUNCTION ASSOCIATED WITH INVASIVE MECHANICAL \\ VENTILATION IN CRITICALLY ILL ADULT PATIENTS \\ DISFUNÇÃO DIAFRAGMÁTICA ASSOCIADA À VENTILAÇÃO MECÂNICA INVASIVA EM \\ PACIENTES ADULTOS CRÍTICOS
}

\section{Pablo Tocalini ${ }^{1}$, Antonela Vicente ${ }^{2}$, Juan Manuel Carballo ${ }^{3}$, Luis Ignacio Garegnani'.}

\begin{abstract}
Las personas internadas en la Unidad de terapia intensiva, muchas veces requieren estar conectadas a un respirador artificial, mientras se resuelve la enfermedad. Sin embargo estar conectados al respirador tiene sus consecuencias, dentro de las cuales se encuentra la disfunción diafragmática inducida por la ventilación mecánica. Esta entidad se caracteriza por afectar al principal músculo de la respiración, el diafragma, generándole debilidad, lo cual tiene impacto significativo a la hora de querer separar al paciente del respirador artificial. El mejor método para diagnosticarla continúa siendo la presión negativa transdiafragmática generada por la estimulación bilateral de los nervios frénicos, aunque por la falta de practicidad, se comenzó a utilizar la ecografía diafragmática. Tanto los datos como las repercusiones de esta patología todavía no son del todo claros.
\end{abstract}

\section{Conceptos clave:}

Que se sabe del tema:

La disfunción diafragmática inducida por la ventilación mecánica es una entidad de reciente definición y de interés creciente, que se caracteriza por la atrofia y la disfunción contráctil del diafragma. El interés sobre esta patología recae en la asociación con resultados clínicos negativos como mayor duración de la VMI, falla en el destete y mayor mortalidad.

Que se aporta con el trabajo:

$\mathrm{Si}$ bien es una patología con impacto clínico significativo sobre los pacientes en VMI, se puede ver que todavía hay heterogeneidad en los valores de referencia y en los métodos diagnósticos utilizados. Así también muchos de los trabajos analizados no estaban diseñados ni potenciados para hallar relación entre la DDIVM y variables clínicas. Todo esto se pone de manifiesto en la variabilidad de los resultados que presentan los trabajos analizados.

1- Licenciado Kinesiólogo Fisiatra. Clínica Basilea, CABA, Argentina 2- Licenciada Kinesióloga Fisiatra. Hospital General de Agudos Parmenio Piñero. CABA, Argentina.

3- Licenciado Kinesiólogo Fisiatra. Hospital General de Agudos Donación Francisco Santojanni. CABA, Argentina.

4- Licenciado Kinesiólogo Fisiatra. Centro Cochrane Asociado Instituto Universitario Hospital Italiano. CABA, Argentina.

5- E-mail de contacto: pablotocalini@hotmail.com

\section{Resumen:}

La ventilación mecánica invasiva (VMI) es una terapia de soporte vital que no está exenta de complicaciones, como la disfunción diafragmática inducida por la ventilación mecánica, que se caracteriza por atrofia y debilidad de dicho musculo. El interés en esta patología parece estar basado en la asociación encontrada con resultados clínicos negativos. La prevalencia oscila entre $29 \%$ y $80 \%$, probablemente debido a la variabilidad entre los diferentes métodos diagnósticos y el momento de la evaluación. El patrón de oro continúa siendo la presión negativa transdiafragmática generada por la estimulación bilateral de los nervios frénicos, aunque por la falta de practicidad, se comenzó a utilizar la ecografía diafragmática. Si bien cuenta con varias modalidades, no todas tienen análisis de correlación con el patrón de oro. Otra problemática surge al momento de analizar la asociación independiente entre la patología y la VMI, ya que los factores de confusión no son sencillos de aislar.

Palabras clave: respiración artificial; unidades de cuidados intensivos; mortalidad; diafragma; epidemiología.

\section{Abstract:}

Invasive mechanical ventilation (VMI) is a life support therapy that is not free of complications, such as ventilator induced diaphragmatic dysfunction, which is characterized by atrophy and weakness of the muscle. Interest in this pathology seems to be based on the association found with negative clinical results. The prevalence ranges between $29 \%$ and $80 \%$, probably due to the variability between the different diagnostic methods and the time of evaluation. The gold standard continues to be the transdiaphragmatic negative pressure generated by the bilateral stimulation of the phrenic nerves, although due to the lack of practicality, diaphragmatic ultrasound began to be used. Although it has several modalities, not all of them have correlation analysis with the gold standard. Another problem arises when analyzing the independent association between pathology and VMI, since confounding factors are not easy to isolate.

Keywords: respiration, artificial; intensive care units; mortality; diaphragm; epidemiology.

\section{Resumo}

A ventilação mecânica invasiva (VMI) é uma terapia de suporte de vida que não apresenta complicações, como disfunção diafragmática induzida por ventilação mecânica, caracterizada por atrofia e fraqueza do referido músculo. O interesse por essa patologia parece basear-se na associação encontrada com resultados clínicos negativos. A prevalência varia de $29 \%$ a $80 \%$, provavelmente devido à variabilidade entre os diferentes métodos de diagnóstico e o tempo de avaliação. O padrão ouro continua sendo a pressão negativa transdiafragmática gerada pela estimulação bilateral dos nervos frênicos, embora, devido à falta de praticidade, o ultra-som diafragmático tenha começado a ser utilizado. Embora tenha várias modalidades, nem todos têm análises de correlação com o padrão-ouro. Outro problema surge ao analisar a associação independente entre patologia e IMV, uma vez que fatores de confusão não são fáceis de isolar.

Palavras-chave: respiração artificial; unidades de terapia intensiva; mortalidade; diafragma; epidemiologia. 


\section{Introducción}

La ventilación mecánica invasiva (VMI) es una terapia de soporte vital, cuya utilización fue en aumento ${ }^{1}$. Alrededor del $40 \%$ de los pacientes requieren ventilación mecánica (VM) durante la internación en la unidad de cuidados intensivos $(\mathrm{UCl})^{2}$ y entre un $10 \%$ al $15 \%$ la requerirán de manera prolongada ${ }^{3}$. Una de las etapas más importantes de su implementación es el destete, que abarca hasta un $40 \%$ del tiempo total de internación en $\mathrm{UCl}^{2,4}$ y en la que se observa que entre un $20 \%$ al $40 \%$ de los pacientes presentan dificultades para interrumpir el uso de esta terapéutica ${ }^{4}$. Esto implica, mayor tiempo en VMI y mayor estadía en $\mathrm{UCl}$, asociándose a mayores $\operatorname{costos}^{1}$ y mayor morbimortalidad ${ }^{1,5}$. Si bien la VMI es una terapéutica de soporte vital, no está exenta de complicaciones ${ }^{6}$, que tienen impacto sobre el destete y pueden comenzar en las primeras horas tras la implementación del soporte ventilatorio ${ }^{7,8}$. Si bien se han descripto múltiples complicaciones relacionadas con la VMI, algunas parecen ser difíciles de reconocer clínicamente, lo cual lleva al subdiagnóstico de entidades ${ }^{9}$, como la atrofia y lesión de las fibras musculares ${ }^{10}$. Esto conduce al desarrollo de debilidad adquirida en la unidad de cuidados intensivos (DAUCI), cuyo principal enfoque es la disfunción de los músculos estriados de las extremidades ${ }^{11}$. El diafragma también se ve afectado, incluso en menor tiempo ${ }^{12}$. Éste es el músculo principal de la ventilación ${ }^{13}$, por lo que su correcto funcionamiento tiene un gran impacto en el destete de la VMI ${ }^{14,15}$.

Durante el período inicial de la enfermedad crítica, uno de los objetivos de la implementación de la VM es la puesta en reposo de los músculos respiratorios, que permanecen en desuso, generando acortamiento pasivo y menor requerimiento energético ${ }^{1}$, pudiendo dar paso a lo que se conoce como disfunción diafragmática inducida por la ventilación mecánica (DDIVM), término inicialmente descripto por Vassilakopoulos y Petrof ${ }^{16}$. Esta entidad puede ser definida como la pérdida de la capacidad de generación de fuerza, por parte del diafragma, relacionada con el uso de la $\mathrm{VM}^{3}$ y se caracteriza por la atrofia y la disfunción contráctil de dicho músculo ${ }^{17}$. La prevalencia reportada oscila entre $29 \%{ }^{13}$ y $80 \%{ }^{18}$ probablemente debido a los diferentes métodos diagnósticos, así como también al momento de la evaluación. El interés sobre las características de esta entidad recae en la asociación con resultados clínicos negativos, como mayor duración de la VMI, falla en el destete y mayor mortalidad ${ }^{19}$.

La medición de la presión negativa transdiafragmática generada por la estimulación bilateral de los nervios frénicos (TwPdi, del inglés "twitch transdiaphragmatic pressure"), continúa siendo el patrón de oro para diagnosticarla, aunque por mayor practicidad de implementación, se comenzó a utilizar la ecografía diafragmática $(E D)^{19,20}$. Si bien esto supone un avance para la detección y el seguimiento, acarrea problemas, ya que solo algunos de los métodos de ED tienen análisis de correlación con el patrón de oro ${ }^{19}$ y tampoco hay un punto de corte establecido de horas de VMI a partir de la cual se considere que la disfunción diafragmática (DD) es generada sólo por la VMI y no por la combinación de otros factores como por ejemplo la presencia de sepsis ${ }^{21}$. Cuando se analiza histológicamente al diafragma, en donantes de órganos, se puede tener cierto control sobre la ausencia de sepsis ${ }^{22}$. Esto se dificulta cuando evalúan poblaciones generales y es aquí donde la asociación independiente entre DD y VMI se vuelve problemática. Es por ello que el objetivo de este trabajo fue revisar la literatura relacionada con la DDIVM, sus características clínicas, sus métodos diagnósticos y su impacto sobre las principales variables de resultado en la $\mathrm{UCl}$.

\section{Materiales y Métodos}

\section{Estrategia de búsqueda}

Se realizó una búsqueda bibliográfica en la base de datos Medline y Cochrane Library el 20 de Febrero de 2019. Debido a la falta de término Mesh (en Pubmed) para DDIVM, se utilizaron los términos "VIDD", "ventilator-induced diaphragm dysfunction", "diaphragmatic injury induced by mechanical ventilation" y "Diaphragm dysfunction" combinados entre sí con el operador booleano "OR" y estos con las palabras claves para VMI "Respiration, Artificial", "Artificial Respiration" y "Mechanical Ventilation". Se aplicó también el truncado de palabra y la búsqueda por título y resumen. A su vez se realizó la búsqueda manual en las referencias bibliográficas de los estudios incluidos $y$ revisiones literarias publicadas.

\section{Criterios de selección}

Se incluyeron aquellos trabajos publicados en inglés y español, realizados en pacientes adultos (mayores de 18 años), internados en $\mathrm{UCl}$, con requerimiento de $\mathrm{VMI}$ vía intubación orotraqueal, que evalúen métodos diagnósticos para DD y/o informen variables de resultado en estos pacientes.

En Medline la búsqueda arrojó 859 trabajos. Luego de revisar por título y resumen se excluyeron 780 , por no tener resultado de interés, por idioma diferente al inglés 0 español, por ser protocolos o por estar realizados en animales o niños. De los restantes, luego de la revisión por texto completo, se seleccionaron 18 estudios para la confección del presente texto, de los cuales 2, fueron incorporados luego de revisar manualmente las referencias bibliográficas (Figura 1). 


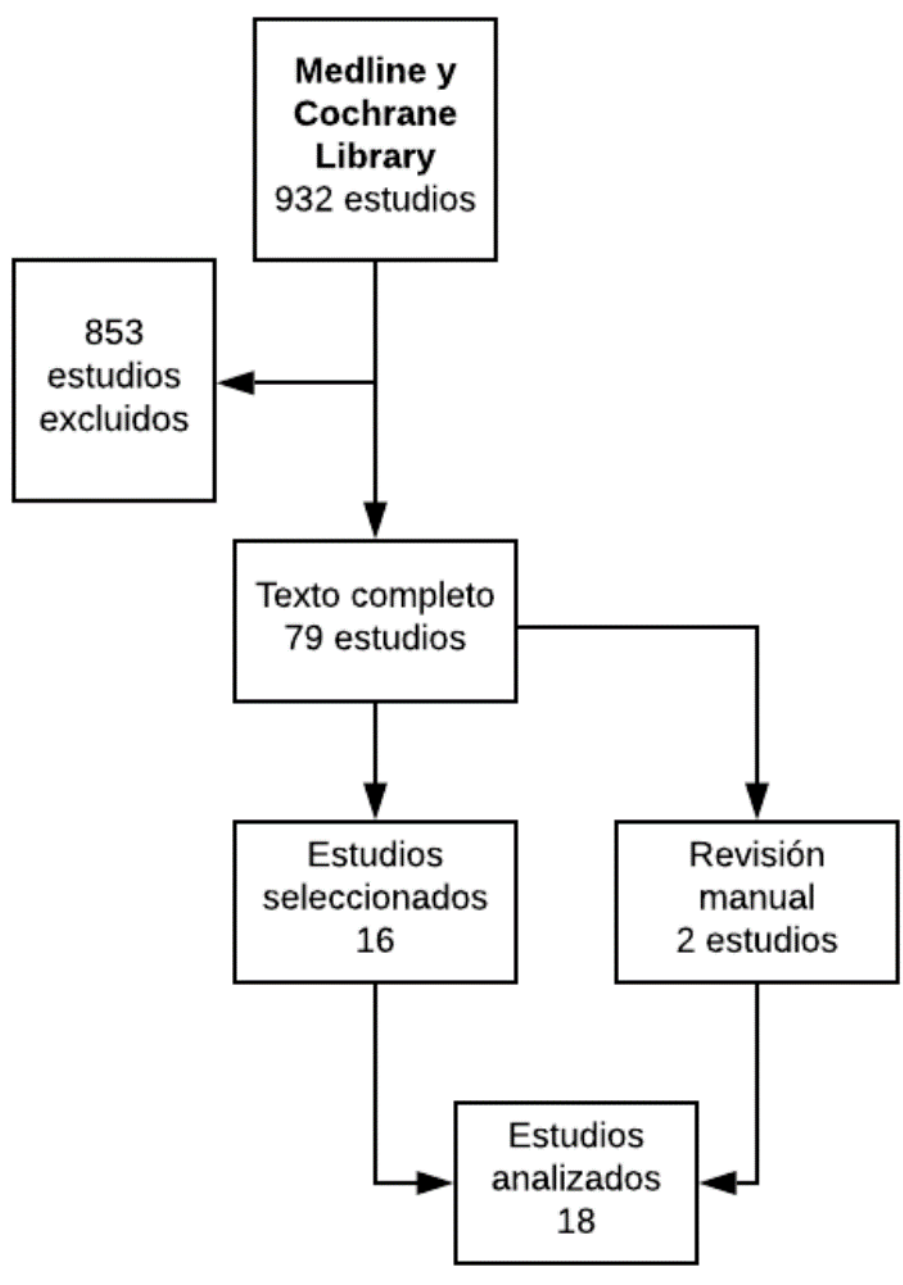

Figura $\mathbf{N}^{\circ}$ 1. Diagrama de Flujo

En Cochrane Library la búsqueda arrojó 73 trabajos. Después de revisar por título y resumen, todos fueron excluidos, por no tener resultado de interés, por idioma diferente al inglés o español, por ser protocolos, por estar realizados en animales o niños o por haber sido incluidos en la búsqueda en Medline.

\section{Desarrollo}

\section{EPIDEMIOLOGÍA}

La prevalencia reportada sobre DDIVM es variable, desde $29 \%{ }^{13}$ a $80 \%{ }^{18}$ (tabla 1 ). Esto podría deberse a la variabilidad en las poblaciones estudiadas, el momento del registro, la herramienta diagnóstica utilizada así como también el punto de corte seleccionado para establecer el diagnóstico y los días en VMI previos a la evaluación. 
Tabla $\mathrm{N}^{\circ}$ 1: Prevalencia según momento de evaluación

\begin{tabular}{|c|c|c|c|c|c|}
\hline $\begin{array}{l}\text { Momento General de } \\
\text { Evaluación }\end{array}$ & $\begin{array}{l}\text { Momento del cálculo de } \\
\text { prevalencia }\end{array}$ & Autor & Método Dg & $\begin{array}{c}\text { Soporte } \\
\text { ventilatorio }\end{array}$ & Prevalencia \\
\hline \multirow{7}{*}{ Día de la PVE } & \multirow{2}{*}{ Antes de la PVE } & $\operatorname{Kim} 2011^{13}$ & EXdi & VMI & $29 \%$ \\
\hline & & Dres $2017^{23}$ & Ptr,Stim & VMI & $63 \%$ \\
\hline & \multirow{4}{*}{ Durante la PVE } & Mariani $2016^{25}$ & EXdi & $\mathrm{T}-\mathrm{T}$ & $38 \%$ \\
\hline & & Jung $2016^{18}$ & Ptr,Stim & VMI (PC-CSV) & $80 \%$ \\
\hline & & Lu $2016^{24}$ & TFdi & VMI (PC-CSV) & $34 \%$ \\
\hline & & Huang $2017^{4}$ & EXdi & $\begin{array}{c}\text { VMI (PC-CSV) o } \\
\text { T-T }\end{array}$ & $75 \%$ \\
\hline & $\begin{array}{c}\text { Luego de la falla de la } \\
\text { PVE }\end{array}$ & Laghi $2003^{26}$ & TwPdi & VMI & $66 \%$ \\
\hline \multirow{3}{*}{$24 \mathrm{hs}$ de IOT+VMI } & \multirow{3}{*}{$24 \mathrm{hs}$} & Demoule $2013^{27}$ & Ptr,Stim & VMI & $64 \%$ \\
\hline & & Demoule $2016^{28}$ & Ptr,Stim & VMI & $53 \%$ \\
\hline & & Dubé $2017^{19}$ & Ptr,Stim & VMI & $77 \%$ \\
\hline \multirow{4}{*}{$>24 \mathrm{hs} \mathrm{IOT}+\mathrm{VMI}$} & A la semana & Goligher $2015^{15}$ & Tdi & VMI & $\begin{array}{c}\text { Cae } 10 \%: \\
44 \%\end{array}$ \\
\hline & $\begin{array}{l}\text { Día de TQT, VE, } \\
\text { Extubación o muerte }\end{array}$ & Schepens $2015^{12}$ & Tdi & - & $\begin{array}{c}\text { Cae } 10 \%: \\
77 \%\end{array}$ \\
\hline & PC-CSV una hora & Dubé $2017^{19}$ & Ptr,Stim & VMI & $68 \%$ \\
\hline & 4 días de VMI & Goligher $2018^{29}$ & Tdi & VMI & $\begin{array}{l}\text { Cae } 10 \%: \\
41 \%\end{array}$ \\
\hline
\end{tabular}

Varios autores informaron la prevalencia en relación al destete. Algunos antes de realizar la primera prueba de ventilación espontánea $(P V E)^{13,23}$, otros durante la realización de la misma ${ }^{4,18,24,25}$ y Laghi et $\mathrm{al}^{26}$ la informó en pacientes que fallan la PVE. A su vez, varios autores la informaron en relación al tiempo transcurrido en VMI $^{12,15,19,27-29}$.

\section{Día de la prueba de ventilación espontánea}

\section{Antes de la PVE}

Dres et $\mathrm{al}^{23}$ observó una prevalencia de $63 \%$ y Kim et $\mathrm{al}^{13}$ del $29 \%$. Esta diferencia puede estar relacionada por un lado, al método diagnostico, ya que Dres et $a^{23}$ utilizó el cambio en la presión a nivel del tubo orotraqueal generada por la estimulación bilateral de los nervios frénicos (Ptr,Stim, del inglés "changes in endotracheal tube pressure induced by bilateral phrenic nerve stimulation during airway occlusion") para la valoración de la DD y Kim et $\mathrm{al}^{13}$ la excursión diafragmática (EXdi, del inglés "diaphragm excursion"), evaluada por ED. Por otro lado, también puede deberse a la población analizada, ya que Kim et al ${ }^{13}$ excluyó a todos los pacientes con DD previa al inicio del estudio.

\section{Durante la PVE}

Mariani et $\mathrm{al}^{25}$ informó un $38 \%$, Huang et $\mathrm{al}^{4}$ un $75 \%$, Lu et $\mathrm{al}^{24}$ un $34 \%$ y Jung et $\mathrm{al}^{18}$ un $80 \%$. Son evidente las diferencias entre los estudios y la comparación resulta dificultosa ya que las poblaciones, la forma de realizar la PVE, el tiempo de duración de la misma, así como también la herramienta diagnóstica difieren entre ellos.

Huang et $\mathrm{al}^{4}$, utilizando la EXdi, incluyó pacientes mayores a 80 años con varias comorbilidades, lo cual podría explicar la elevada tasa de DD. Jung et $\mathrm{al}^{18}$, utilizando Ptr,Stim, también informó una tasa elevada de DD, la cual se puede explicar porque incluyó sólo pacientes con diagnóstico previo de DAUCl y la prevalencia se calculó a los 60 minutos de comenzada la PVE. Al analizar el trabajo de Lu et $\mathrm{al}^{24}$, quien utilizó la fracción de engrosamiento diafragmático (TFdi, del inglés "diaphragm thickening fraction") para valorar la DD, observamos que si bien eran pacientes que cumplían con los criterios de destete prolongado y se podría considerar que tienen mayor debilidad muscular, se excluyó a los pacientes con DD previa. Además, el punto de corte que consideró para el diagnóstico, era menor al que según Dubé et $a^{19}$ tiene buena correlación con Ptr,Stim. Esto podría explicar la menor tasa de DD. Algo parecido sucedió en el trabajo de 
Mariani et $\mathrm{al}^{25}$. Solo incluyó pacientes con más de 7 días de VMI, que completaran exitosamente la PVE, tal vez excluyendo a un gran número de pacientes que no lograron completar la PVE pero que sí podrían tener DD.

\section{Después de la PVE}

Laghi et $a^{26}$ informó una prevalencia de $66 \%$ en pacientes que fallaban la PVE, siendo el único autor que la informó en este momento.

A las 24 horas de intubación orotraqueal y ventilación mecánica invasiva

Demoule et al, en $2013^{27}$ y $2016^{28}$, y Dubé et al ${ }^{19}$ en 2017 informaron la incidencia de DD a las 24 hs de VMI, reportando $64 \%, 53 \%$ y $77 \%$ respectivamente. La edad y el score de gravedad en los tres trabajos eran similares. Si bien los pacientes fueron evaluados dentro de las primeras 24 hs de $\mathrm{VMI}$, hay evidencia que el diafragma muestra signos de lesión en tan solo 18 hs de $\mathrm{VMI}^{22}$, por lo que esta disfunción podría ser causa de ella, aunque también de la sepsis al ingreso a la $\mathrm{UCl}$, debido a la alta prevalencia reportada $^{19,27}$

Demoule et al, explicó la diferencia entre sus cohortes, basándose en la del 2016, si bien fueron evaluados a las 24 hs de VMI, sólo se incluyeron pacientes con más de 5 días de VMI, por lo que los pacientes más graves probablemente podrían haber muerto antes de ser incluidos y por eso la incidencia de DD puede ser menor ${ }^{27,28}$.
Más de 24 horas de intubación orotraqueal y ventilación mecánica invasiva

Grosu et al $^{3}$ informó, utilizando como diagnóstico el engrosamiento diafragmático ( $\mathrm{Tdi}$, del inglés "diaphragm thickness"), que a las 72 hs disminuyó en el $84 \%$ de los pacientes. Sin embargo no informó el porcentaje de cambio en el grosor respecto del basal, lo cual lo vuelve incomparable con las otras cohortes.

Goligher et al, en $2015^{15}$ y $2018^{29}$ consideró un Tdi menor al $10 \%$ del basal como punto de corte para determinar DD. En el $2018^{29}$ informó que el Tdi disminuía al menos un $10 \%$ en el $41 \%$ de los pacientes y $44 \%$ en la cohorte del $2015^{15}$. La diferencia podría estar relacionada al momento de reevaluación, ya que la prevalencia en el estudio de 2018 se informó a los 4 días de VMI y en el 2015 a la semana ${ }^{15,29}$, ya que la edad y el score de gravedad en ambas fueron similares. Schepens et al $^{12}$ informó una caída del Tdi de al menos $10 \%$ en el $77 \%$ de los pacientes en el día de la traqueostomía (TQT), extubación, retorno a la ventilación espontánea sin asistencia o muerte. La diferencia es notable con respecto a las cohortes de Goligher et $\mathrm{al}^{15,29}$, sin embargo Schepens et al no informa los días de VMI previos al momento de la reevaluación y la media de SAPS II fue superior a lo reportado por otros autores ${ }^{12,15,29}$.

\section{DIAGNÓSTICO}

Se han utilizado varios métodos (invasivos y no invasivos) para evaluar la fuerza y/o función del diafragma y llegar así al diagnóstico de DD (tabla 2).

Tabla $\mathrm{N}^{\circ}$ 2: métodos diagnósticos invasivos y no invasivos.

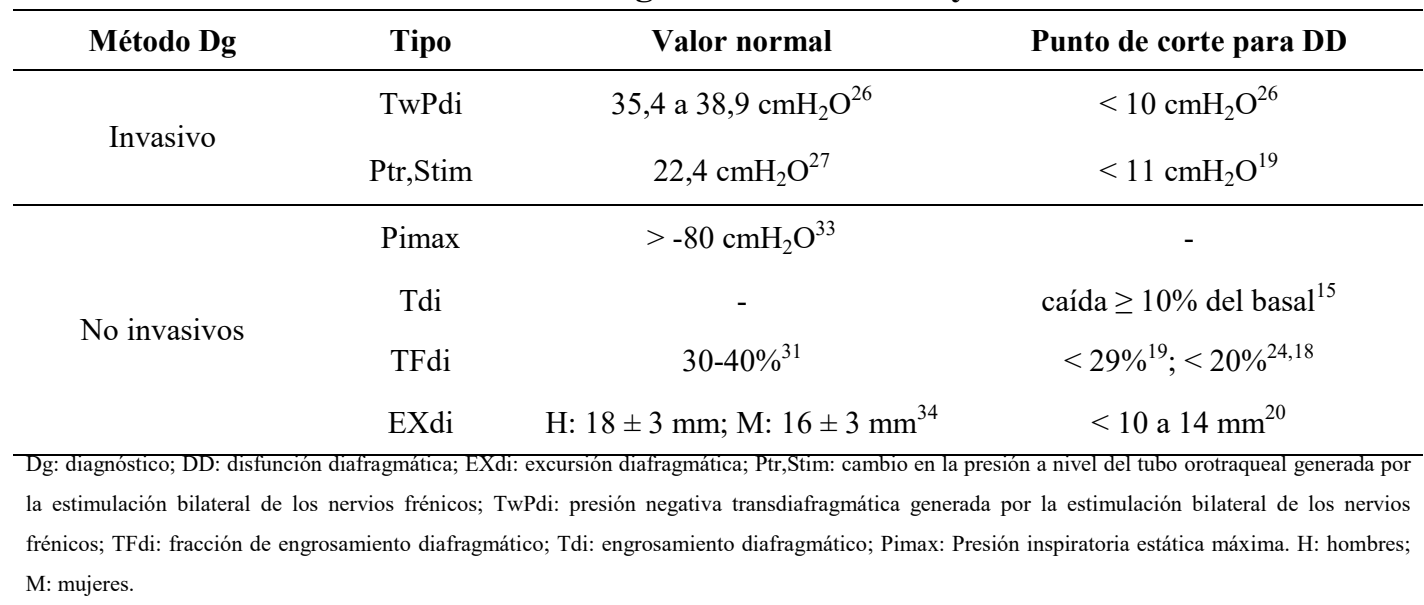

\section{Métodos invasivos}

Medición de la presión negativa transdiafragmática generada por la estimulación bilateral de los nervios frénicos (TwPdi)

El patrón de oro para valorar la fuerza diafragmática es la $\mathrm{TwPdi}^{30}$, que requiere de un catéter esofágico y uno gástrico más los elementos de estimulación nerviosa. Es un método que requiere personal calificado, consume tiempo y no es práctico para el uso diario ${ }^{19,31}$. Se calcula con la fórmula TwPdi: $\Delta$ presión gástrica - $\Delta$ presión esofágica $(\Delta$ : diferencia entre la presión previa a la estimulación y el pico de presión alcanzado luego de la estimulación) ${ }^{9}$.

Esta técnica fue utilizada por primera vez por Watson et al $^{30}$ en pacientes internados en $\mathrm{UCl}$, desarrollando una técnica que podía ser aplicada sin la necesidad de esfuerzo voluntario y por lo tanto en pacientes sedados. Más allá de 
la dificultad práctica, algunos estudios la utilizaron como método $^{26,32}$. Según Laghi et $\mathrm{al}^{26}$ el valor normal de en sujetos sanos va de 35.4 a $38.9 \mathrm{cmH}_{2} \mathrm{O}$ mientras que en su cohorte aquellos que fallaron el destete tenían menos de 10 $\mathrm{cmH}_{2} \mathrm{O}$, similar a lo encontrado por Hermans et $\mathrm{al}^{32}$.

Medición del cambio en la presión a nivel del tubo orotraqueal generada por la estimulación bilateral de los nervios frénicos (Ptr, Stim)

También utilizada por primera vez por Watson et $\mathrm{al}^{30}$, quien intentó correlacionarlo con la Ptr,Stim, encontrando correlación positiva muy fuerte, por lo que en varios trabajos posteriores fue considerado como el patrón de oro ${ }^{19,27}$. Si bien no requiere del balón gástrico y esofágico, si necesita del instrumental para la estimulación eléctrica, ya que se calcula como el diferencial de presión a nivel del tubo orotraqueal antes y después de la estimulación del nervio frénico ${ }^{30}$. Los estudios que la utilizan toman un valor menor a $11 \mathrm{cmH}_{2} \mathrm{O}$ para determinar $D D^{18,19,23,27,28}$, basándose en la recomendación de la Sociedad americana de tórax y Sociedad europea respiratoria ${ }^{33}$. Este valor fue validado por Demoule et $\mathrm{al}^{27}$ en una población de pacientes anestesiados por un período corto de tiempo para la realización de cirugías electivas.

\section{Métodos no invasivos}

\section{Presión inspiratoria estática máxima}

Es una forma simple, rápida y fácil de medir la fuerza de los músculos inspiratorios ${ }^{33}$ y aunque mostró tener una buena correlación con la $\mathrm{Ptr}, \mathrm{Stim}^{18}$, no evalúa al diafragma de forma aislada ${ }^{33}$, sino que debe interpretarse como una evaluación global de los músculos inspiratorios ${ }^{18}$, motivo por el cual no es utilizada por los estudios para el diagnóstico de DD.

\section{Ecografía diafragmática (ED)}

La evaluación al lado de la cama del diafragma no es algo fácil $^{31}$. La ED es una técnica precisa, segura y fácil de implementar al lado de la cama y hace unos años se ha comenzado a utilizar para la evaluación del diafragma en la $\mathrm{UCl}^{20,34}$. Si bien tiene limitaciones, como la dificultad para encontrar una buena ventana para evaluar, mostró tener buena correlación intra e inter-observador ${ }^{34,35}$ y puede ser utilizada para la evaluación funcional y morfológica del diafragma e incluso puede implementarse de manera práctica para repetir mediciones a lo largo del tiempo ${ }^{20}$ y valorar diversos parámetros de la actividad diafragmática.

\section{Engrosamiento diafragmático (Tdi)}

Se define como la distancia desde la parte media de la pleura diafragmática hasta la parte media de la pleura peritoneal evaluado al final de la espiración en la zona de aposición del diafragma ${ }^{2,10,34}$. Baldwin et $\mathrm{al}^{36}$ hace referencia que esta técnica es precisa, fácil de realizar y que es fiable para la medición en pacientes en posición reclinada. Según Goligher et $\mathrm{al}^{31}$ el Tdi puede utilizarse para evaluar la variación del engrosamiento del diafragma en pacientes con VMI, por lo que podría detectar fiablemente atrofia diafragmática y evaluar la función contráctil sin que ese engrosamiento refleje el inflado pasivo del tórax. Sin embargo Dubé et $\mathrm{al}^{19}$, pone en duda esta utilidad del Tdi y sugiere no utilizarlo como estimador de la contracción de este músculo, por lo menos en el ámbito de la $\mathrm{UCl}$, al encontrar correlación débil con la Ptr,Stim. También refiere que el aumento del grosor podría representar un problema patológico y no un incremento de la masa muscular funcional. La mayoría de los autores que utilizan el Tdi, informan que un descenso en el engrosamiento con respecto al basal se relaciona con $D D^{2,3,12,15,29}$ e incluso algunos ${ }^{15,29}$ utilizan como punto de corte un descenso del Tdi mayor o igual al $10 \%$ del basal como determinante de esto.

\section{Fracción de engrosamiento diafragmático (TFdi)}

Se calcula utilizando el ecógrafo en modo $M$ para obtener las variables y aplicarlas en la fórmula: TFdi: (Grosor al final de la inspiración - Grosor al final de la espiración)/ Grosor al final de la espiración ${ }^{34}$. El valor normal en voluntarios sanos oscila entre $30-40 \%{ }^{31}$. Goligher ${ }^{31}$ plantea utilizarla solo para la valoración cualitativa de la actividad muscular inspiratoria. Sin embargo Dubé et al $^{19}$, demuestra que hay correlación positiva muy fuerte entre la TFdi y Ptr,Stim y que puede detectar DD en el momento que el paciente tolera PC-CSV. Con respecto a los estudios que lo toman en cuenta $^{7,10,15,18,19,23,24}$, algunos establecen puntos de corte para determinar el diagnóstico de DD.

Lu et $\mathrm{al}^{24}$ y Jung et $\mathrm{al}^{18}$ establecen como punto de corte una TFdi $<20 \%$ y Zambon et al $^{10}$ lo utiliza como variable para evaluar la actividad diafragmática en pacientes con diferentes niveles de soporte ventilatorio. Por su parte Dres et al, en el $2017^{23}$ y $2018^{7}$ utiliza a la TFdi como variable para valorar la asociación con resultados clínicos, encontrando que una TFdi $<25.8 \%$ es buen predictor de falla en el destete ${ }^{7}$.

\section{Excursión diafragmática (EXdi)}

La utilización de la EXdi como índice de la función diafragmática debe ser limitada a pacientes que ventilen espontáneamente. Para evaluarla es mejor utilizar el modo ecográfico $\mathrm{M}^{20}$.

El valor normal en pacientes sanos es de $18 \pm 3 \mathrm{~mm}$ en hombres y $16 \pm 3 \mathrm{~mm}$ en mujeres ${ }^{34}$ mientras que para diagnosticar DD los puntos de corte reportados van de 10 a $14 \mathrm{~mm}$ en ventilación espontánea tranquila ${ }^{20}$. Según Dubé et al $^{19}$ la EXdi depende del grado de contracción activa y desplazamiento pasivo del diafragma generado por el ventilador. Kim et $\mathrm{al}^{13}$ toma un punto de corte menor a 10 $\mathrm{mm}$ para diagnosticar DD, el cual fue algo arbitrario ya que no había hasta ese momento valores normales en pacientes ventilados.

Dres et $a^{23}$ evalúa la EXdi previo a la realización de la PVE sin establecer un punto de corte para detectar DD, sino que intenta ver si se comporta como predictor de destete. Dubé et $\mathrm{al}^{19}$ reporta correlación débil entre Ptr,Stim y EXdi al inicio de la VMI (evaluación dentro de las 24 hs de VMI) y correlación media entre las mismas variables al momento que el paciente tolera PC-CSV una hora. Al mismo tiempo, evalua la precisión diagnóstica del Exdi para DD y encuentra que tanto al inicio de la VMI como al momento de pasar a PC-CSV no puede identificar de forma fiable la $D D^{19}$.

\section{VARIABLES DE RESULTADO}

Con el objetivo de analizar el impacto de la DD sobre las distintas variables de resultados de los pacientes, se seleccionaron los trabajos que describen dichas variables (tabla 3). 
Tabla N³: Resumen de los trabajos incluidos

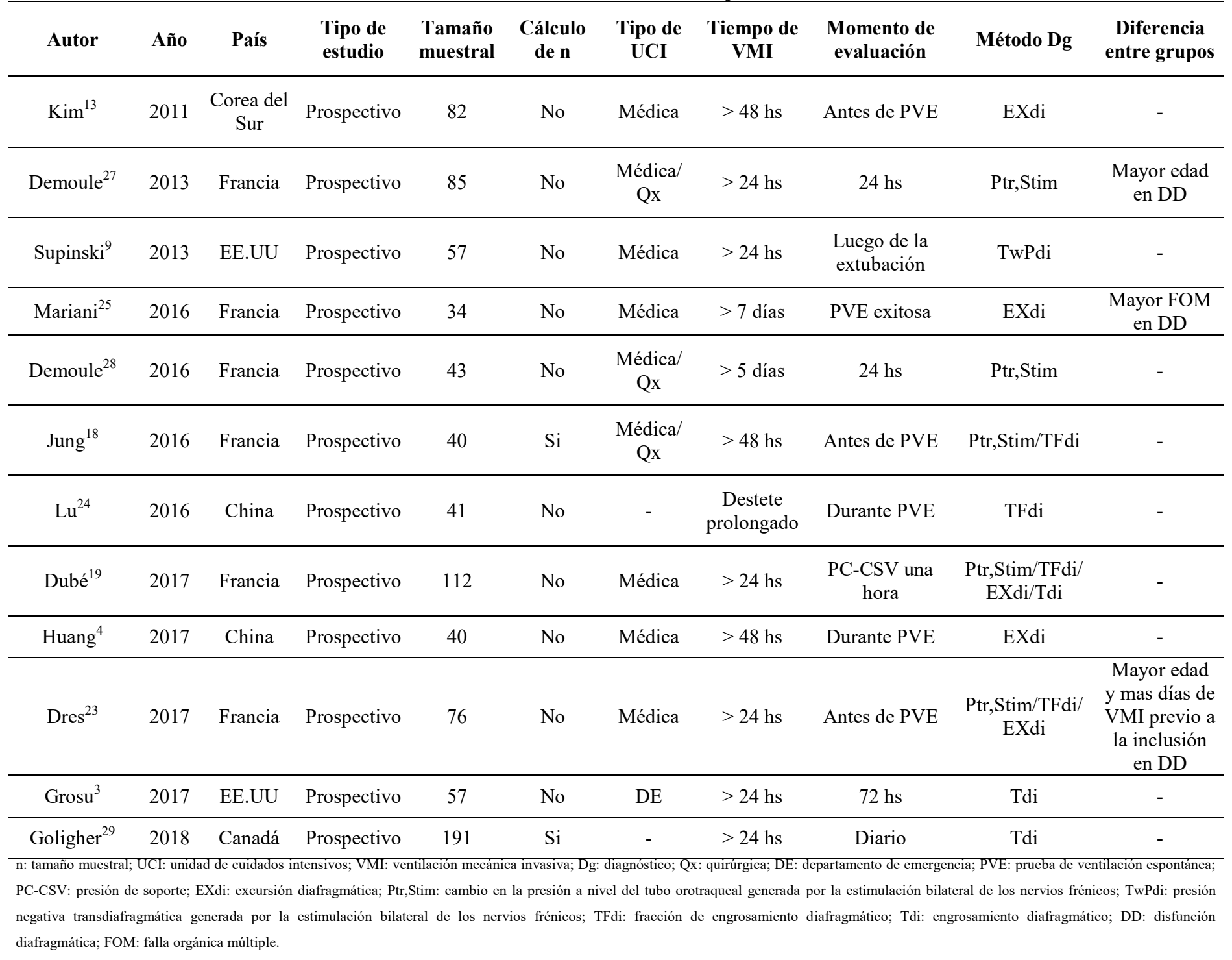

Kim et $\mathrm{al}^{13}$ encontró que los pacientes con DD tenían mayor tasa de falla primaria y secundaria de destete, así como también mayor cantidad de horas de VMI, mayor tiempo de destete, mayor estadía en UTI y hospitalaria. Sin embargo, no encontró diferencia en cuanto a la mortalidad hospitalaria entre los pacientes con DD y aquellos sin DD. Los pacientes no tenían diferencia en cuanto a la edad entre los grupos, pero no informaron ningún score de gravedad, que nos permita tener una idea del estado crítico de los pacientes evaluados.

Demoule et $\mathrm{al}^{27}$ encontró que los pacientes con DD a las 24 hs de ingreso presentaban mayor mortalidad tanto en $\mathrm{UCl}$ como en el hospital, sin diferencias entre los grupos en cuanto a duración de la VMI, tasa de TQT y estadía tanto en $\mathrm{UCl}$ como en el hospital. Los pacientes con DD eran mayores, tenían mayor prevalencia de sepsis al ingreso a la $\mathrm{UCl}$ y estaban más graves que aquellos que no tenían DD, estas últimas dos variables con diferencias estadísticamente significativas entre los grupos. En base a esto se plantea si la DD podría ser una expresión más de la falla multiorgánica. Con respecto a la influencia de la VMI sobre la $\mathrm{DD}$, si bien el tiempo de evaluación fue corto, no pueden descartar su influencia sobre la DD. No hay que dejar de lado que queda por establecer si existe relación causal entre la mortalidad y la DD ya que el estudio no estaba potenciado con tal objetivo.

Supinski et al ${ }^{9}$ encontró que los pacientes con una TwPdi < $10 \mathrm{cmH}_{2} \mathrm{O}$ tenían mayor mortalidad en $\mathrm{UCl}$, requerían más del doble de tiempo para el destete y mayor duración de VMI posterior a la evaluación con respecto a los que tenían una TwPdi $\geq 10 \mathrm{cmH}_{2} \mathrm{O}$. Esta diferencia parecería deberse solo a la debilidad diafragmática ya que los score de gravedad eran similares entre los dos grupos. Sin embargo, no encontraron asociación entre el valor de TwPdi y los días de VMI previo a la evaluación. A su vez los pacientes sin evidencia de infección tenían valores más bajos de TwPdi que los pacientes sanos $\left(13 \mathrm{cmH}_{2} \mathrm{O} V s 30 \mathrm{cmH}_{2} \mathrm{O}\right.$ ), lo que podría deberse a la acción de la VMI.

Mariani et $a^{25}$ encontró que los pacientes con DD tenían mayor mortalidad en $\mathrm{UCl}$ respecto al subgrupo sin $\mathrm{DD}$, pero no así con respecto a la mortalidad hospitalaria (1 de $21 \mathrm{Vs}$ 
4 de 13). En relación a los pacientes con DD bilateral se observó una tendencia a mayor duración de VMI y estadía en UCI. Ninguno de los pacientes con DD falló la extubación, sin embargo, hay que interpretar con cautela este dato, dado que el estudio no estaba potenciado para tal fin. Con respecto a las variables demográficas, no encontraron diferencia en cuanto a la edad entre los pacientes sin DD y aquellos con DD unilateral y solo una tendencia a mayor edad con respecto a los pacientes con DD bilateral. Si hallaron diferencia en cuanto a la falla multiorgánica, ya que la desarrollaban el $100 \%$ de los pacientes con DD bilateral y solo el $52 \%$ sin DD.

Demoule et $\mathrm{al}^{28}$ encontró que los pacientes con DD presentaban mayor mortalidad en $\mathrm{UCl}$, no encontrando diferencias en cuanto a duración de VMI, mortalidad hospitalaria, estadía en UCl y en el hospital. Con respecto a las variables demográficas, los grupos no diferían en cuanto a la edad, SAPS II ni porcentaje de sepsis en la admisión a UCl.

Jung et $\mathrm{al}^{18}$ encontró que la TFdi se asociaba con éxito de extubación. Sin embargo, este fue un objetivo secundario y el trabajo estaba potenciado para predecir factores de riesgo asociados independientemente al éxito o falla de extubación. Cabe destacar que todos los pacientes con falla en el destete tenían una Ptr,Stim por debajo del punto de corte que usaron para diagnosticar DD, aunque algunos pacientes del grupo de destete exitoso también. Además hay que tener en cuenta que todos los pacientes debían tener diagnóstico de DAUCI previo al ingreso al estudio.

Lu et $\mathrm{al}^{24}$ observó que los pacientes con DD tenían mayor duración de VMI luego de la inclusión, mayor estadía en UCI y mayor mortalidad hospitalaria. A su vez encontró una tendencia a una mayor estadía hospitalaria, aunque sin significancia estadística.

Dubé et $\mathrm{al}^{19}$ encontró que los pacientes con DD presentaron mayor estadía en UCl, más días de VMI y de VMI después de la medición, así como también mayor mortalidad tanto en $\mathrm{UCl}$ como hospitalaria. A su vez hicieron hincapié en el cuidado de la generalización de los resultados, producto que el estudio, si bien tenía una gran muestra, fue realizado sólo en una UCI médica.

Huang et $\mathrm{al}^{4}$ encontró que los pacientes con DD tenían mayor falla de destete, mayor tiempo de destete y mayor tiempo total de VMI. Encontró también una tendencia a mayor estadía tanto en $\mathrm{UCl}$ como en el hospital (aunque sin diferencia estadísticamente significativa) en el grupo con DD y no encontró diferencia con respecto a la mortalidad a 28 días. Sin embargo, como limitación nombra que la muestra era pequeña y que no hay punto de corte establecido para EXdi en pacientes mayores por lo que la elección tal vez no fuera la ideal. Sumado a esto el estudio no estaba potenciado para esta variable en cuestión.

Grosu et $\mathrm{al}^{3}$ encontró que los pacientes desarrollaban adelgazamiento del diafragma a las 72 de VMI y que llamativamente estos presentaban menor tasa de falla de extubación. Sin embargo, la muestra era pequeña y el estudio no estaba potenciado para valorar la correlación entre el adelgazamiento y la fuerza del diafragma. Con respecto a la herramienta de evaluación, no parecería ser la correcta para valorar la fuerza diafragmática, lo cual se vio en el trabajo de Dubé et $\mathrm{al}^{19}$, donde no se observó correlación entre Tdi y el patrón de oro. Por todos estos motivos concluyen que no pueden encontrar una correlación con la falla de extubación.

Dres et $\mathrm{al}^{23}$ encontró que los pacientes con DD eran más grandes, tenían más días de VMI previo a la inclusión en el estudio que los pacientes sin DD. Además, encontró que la DD estaba asociada de manera independiente con la falla de destete. También informó que aquellos pacientes que tenían DD presentaban mayor mortalidad tanto en $\mathrm{UCl}$, como en el hospital, mayor porcentaje de destete dificultoso y prolongado, así como también mayor duración de VMI y más días de estadía en UCl.

Goligher et $\mathrm{al}^{29}$ encontró que el desarrollo de atrofia diafragmática generaba mayor duración de VMI, mayor estadía en $\mathrm{UCl}$ y en el hospital, así como también mayores complicaciones, tasa de re-intubación y TQT que en el subgrupo que no desarrolla atrofia.

\section{Mortalidad}

La mayoría de los trabajos encontraron que los pacientes con DD presentaban mayor mortalidad en $\mathrm{UCl}$ con diferencias estadísticamente significativas con respecto a los pacientes que no tenían $D^{9,19,23,25,27,28}$. Lo mismo sucede con la mortalidad hospitalaria, donde la mayoría de los trabajos encontraron diferencias significativas entre los subgrupos con y sin $\mathrm{DD}^{19,23,24,27}$. Goligher et $\mathrm{al}^{29}$ por su parte, encontró una tendencia a mayor mortalidad tanto en $\mathrm{UCl}$ como en el hospital en los pacientes con DD.

\section{Destete y re-intubación}

Con respecto al destete, la mayoría de los trabajos encontraron mayor falla en el subgrupo con $\mathrm{DD}^{4,13,23}$, así como también mayor tiempo ${ }^{4,9,13}$ y mayor porcentaje de destete dificultoso y prolongado ${ }^{23}$. Por último, Goligher et $a^{29}$ informó mayor tasa de re-intubación en los pacientes con DD, también con diferencia estadísticamente significativa.

\section{Estadía en UCl y hospitalaria}

La mayoría de los estudios encontraron mayor estadía en $\mathrm{UCl}$ en los pacientes con $\mathrm{DD}^{4,13,19,23-25,28,29}$, aunque sin diferencias estadisticamente significativas en tres de ellos $^{4,25,28}$. Goligher et $\mathrm{al}^{29}$ y Kim et $\mathrm{al}^{13}$ encontraron que los pacientes con DD presentaban también mayor estadía hospitalaria, Dres et $\mathrm{al}^{23}$, Lu et $\mathrm{al}^{24}$ y Huang et $\mathrm{al}^{4}$ solo una tendencia a este resultado y Demoule et al en sus dos cohortes no encontró diferencias entre los pacientes con DD y aquellos $\sin D D^{27,28}$.

\section{Duración de VMI}

La mayoría de los estudios encontraron mayor duración de VMI en los pacientes con DD $4,9,13,19,23,24,29$, con diferencias estadísticamente significativas en relación a los pacientes $\sin \mathrm{DD}$, mientras que Mariani et $\mathrm{al}^{25}$ y Demoule et $\mathrm{al}^{28}$ solo encontraron una tendencia a mayor duración de VMI en este subgrupo de pacientes.

Tasa de traqueostomía

Solo dos trabajos informaron esta variable ${ }^{27,29}$. Demoule et $\mathrm{al}^{27}$ no encontró diferencia entre los pacientes con DD y sin $\mathrm{DD}$, mientras que Goligher et $\mathrm{al}^{29}$ encontró que la DD se asociaba de manera independiente con mayor tasa de TQT. 


\section{Conclusión}

La DDIVM es una entidad de reciente definición y de interés creciente, evidenciado por la gran cantidad de publicaciones relacionadas con el tema. Sin embargo, existe amplia variabilidad en los puntos de corte para determinar la alteración y los métodos diagnósticos utilizados. Esto impacta directamente en los resultados que los trabajos informan.

En 2004, se establece inicialmente su definición, la cual es fisiopatológica. Esto podría ser uno de los orígenes de la variabilidad posterior, debido a que la falta de especificidad que conlleva este tipo de definiciones, impide lograr un consenso entre los distintos autores. Inicialmente vemos el impacto a la hora de analizar los criterios de inclusión de los estudios, donde se observa que el tiempo de VMI previo a la inclusión de los participantes es ampliamente variable. Los métodos diagnósticos, no son ajenos a este problema, y esto queda claro al ver los diferentes métodos utilizados, independientemente de la existencia de un patrón de oro. La justificación radica en la dificultad tanto para la implementación clínica inicial del patrón de oro, así como también para el seguimiento posterior. Sin embargo son pocos los estudios que realizan análisis de correlación de los métodos diagnósticos alternativos, aunque son varios los que lo utilizan. Con respecto a los puntos de corte seleccionados para determinar la presencia de la alteración, también es variable entre los distintos trabajos. Estos son los motivos que podrían explicar el amplio rango de prevalencia reportado por los diferentes autores, que también va a influir en las variables de resultado.

A pesar de la consistencia entre estudios respecto de la relación entre DD y la mayoría de las variables de resultado, cabe destacar que varios de estos estudios no fueron diseñados ni potenciados para hallar estas diferencias, por lo que se requieren de más estudios específicamente diseñados para tal objetivo, considerando las posibles variables de confusión que puedan presentarse. Esta imposibilidad de los estudios de encontrar asociación independiente, es lo que probablemente genere que se hable de disfunción diafragmática y no de disfunción diafragmática inducida por la ventilación mecánica.

\section{Limitaciones de responsabilidad:}

la responsabilidad del trabajo es solo de los autores.

\section{Fuentes de apoyo:}

no se recibió apoyo de ningún tipo para la realización de este trabajo.

\section{Originalidad:}

Este manuscrito no ha sido publicado y no está en consideración para su publicación en otro lugar.

\section{Cesión de derechos:}

Los participantes de este trabajo ceden el derecho de autor a la Universidad Nacional de Córdoba para publicar en la Revista de la Facultad de Ciencias Médicas y realizar las traducciones necesarias al idioma inglés.

\section{Conflicto de interés:}

Se declara que ninguno de los autores mencionados previamente presenta conflictos de interés en este estudio.

\section{Agradecimientos:}

Licenciada Kinesióloga Fisiatra Eliana Perez Calvo.

Licenciado Kinesiólogo Fisiatra Sacha Alexis Virgilio.

\section{Bibliografía}

1. Picard M, Jung B, Liang F, Azuelos I, Hussain S, Goldberg $P$, Godin R, Danialou G, Chaturvedi R, Rygiel K, Matecki S, Jaber S, Des Rosiers C, Karpati G, Ferri L, Burelle Y, Turnbull DM, Taivassalo T, Petrof BJ. Mitochondrial dysfunction and lipid accumulation in the human diaphragm during mechanical ventilation. Am J Respir Crit Care Med. 2012 Dec 1;186(11):11409. doi: 10.1164/rccm.201206-0982OC.

2. Grosu HB, Lee YI, Lee J, Eden E, Eikermann M, Rose KM. Diaphragm muscle thinning in patients who are mechanically ventilated. Chest. 2012 Dec;142(6):1455-1460. doi: 10.1378/chest.11-1638.

3. Grosu HB, Ost DE, Lee YI, Song J, Li L, Eden E, Rose $K$. Diaphragm Muscle Thinning in Subjects Receiving Mechanical Ventilation and Its Effect on Extubation. Respir Care. 2017 Jul;62(7):904-911. doi: 10.4187/respcare.05370.

4. Huang $D$, Ma $H$, Zhong W, Wang $X$, Wu $Y$, Qin T, Wang S, Tan $N$. Using M-mode ultrasonography to assess diaphragm dysfunction and predict the success of mechanical ventilation weaning in elderly patients. J Thorac Dis. 2017 Sep;9(9):31773186. doi: 10.21037/jtd.2017.08.16.

5. Esteban A, Frutos-Vivar F, Muriel A, Ferguson ND, Peñuelas $O$, Abraira V, Raymondos $K$, Rios $F$, Nin N, Apezteguía $C$, Violi $D A$, Thille AW, Brochard L, González M, Villagomez AJ, Hurtado J, Davies AR, Du B, Maggiore SM, Pelosi P, Soto L, Tomicic V, $D$ 'Empaire $G$, Matamis $D$, Abroug $F$, Moreno RP, Soares $M A$, Arabi $Y$, Sandi $F$, Jibaja M, Amin $P$, Koh $Y$, Kuiper MA, Bülow HH, Zeggwagh AA, Anzueto A. Evolution of mortality over time in patients receiving mechanical ventilation. Am J Respir Crit Care Med. 2013 Jul 15;188(2):220-30. doi: 10.1164/rccm.201212$21690 \mathrm{C}$

6. Hussain SNA, Cornachione AS, Guichon C, et al. Prolonged controlled mechanical ventilation in humans triggers myofibrillar contractile dysfunction and myofilament protein loss in the diaphragm. Thorax. 2016;71(5):436-445.

7. Dres M, Goligher EC, Dubé BP, Morawiec E, Dangers L, Reuter $D$, Mayaux J, Similowski T, Demoule A. Diaphragm function and weaning from mechanical ventilation: an ultrasound and phrenic nerve stimulation clinical study. Ann Intensive Care. 2018 Apr 23;8(1):53. doi: 10.1186/s13613-018-0401-y.

8. Mankowski RT, Ahmed S, Beaver T, Dirain M, Han C, Hess $P$, Martin T, Smith BK, Someya S, Leeuwenburgh C, Martin $A D$. Intraoperative hemidiaphragm electrical stimulation reduces oxidative stress and upregulates autophagy in surgery patients undergoing mechanical ventilation: exploratory study. J Transl Med. 2016 Oct 26;14(1):305. doi: 10.1186/s12967-016-1060-0.

9. Supinski GS, Callahan LA. Diaphragm weakness in mechanically ventilated critically ill patients. Crit Care. 2013 Jun 20;17(3):R120. doi: $10.1186 /$ cc12792.

10. Zambon M, Beccaria $P$, Matsuno J, Gemma M, Frati $E$, Colombo S, Cabrini L, Landoni G, Zangrillo A. Mechanical Ventilation and Diaphragmatic Atrophy in Critically III Patients: An Ultrasound Study. Crit Care Med. 2016 Jul;44(7):1347-52. doi: 10.1097/CCM.0000000000001657.

11. De Jonghe B, Sharshar T, Lefaucheur JP, Authier FJ, DurandZaleski I, Boussarsar M, Cerf C, Renaud E, Mesrati F, Carlet J, Raphaël JC, Outin H, Bastuji-Garin S; Groupe de Réflexion et d'Etude des Neuromyopathies en Réanimation. Paresis acquired in the intensive care unit: a prospective multicenter study. JAMA. 2002 Dec 11;288(22):2859-67. doi: 10.1001/jama.288.22.2859.

12. Schepens $T$, Verbrugghe $W$, Dams $K$, Corthouts $B$, Parizel PM, Jorens $P G$. The course of diaphragm atrophy in ventilated patients assessed with ultrasound: a longitudinal cohort study. Crit Care. 2015 Dec 7;19:422. doi: 10.1186/s13054-015-1141-0. 
13. Kim WY, Suh HJ, Hong SB, Koh Y, Lim CM. Diaphragm dysfunction assessed by ultrasonography: influence on weaning from mechanical ventilation. Crit Care Med. 2011 Dec;39(12):2627-30. doi: 10.1097/CCM.0b013e3182266408.

14. Jaber S, Petrof BJ, Jung B, Chanques G, Berthet JP, Rabuel C, Bouyabrine $H$, Courouble $P$, Koechlin-Ramonatxo $C$, Sebbane $M$, Similowski T, Scheuermann V, Mebazaa A, Capdevila X, Mornet D, Mercier J, Lacampagne A, Philips A, Matecki S. Rapidly progressive diaphragmatic weakness and injury during mechanical ventilation in humans. Am J Respir Crit Care Med. 2011 Feb 1;183(3):364-71. doi: 10.1164/rccm.201004-06700C.

15. Goligher EC, Fan E, Herridge MS, Murray A, Vorona S, Brace $D$, Rittayamai $N$, Lanys A, Tomlinson G, Singh JM, Bolz SS, Rubenfeld GD, Kavanagh BP, Brochard LJ, Ferguson ND. Evolution of Diaphragm Thickness during Mechanical Ventilation. Impact of Inspiratory Effort. Am J Respir Crit Care Med. 2015 Nov 1;192(9):1080-8. doi: 10.1164/rccm.201503-06200C.

16. Vassilakopoulos $T$, Petrof BJ. Ventilator-induced diaphragmatic dysfunction. Am J Respir Crit Care Med. 2004 Feb 1;169(3):33641. doi: $10.1164 / \mathrm{rccm}$.200304-489CP.

17. Kim WY, Park SH, Kim WY, Huh JW, Hong SB, Koh Y, Lim CM. Effect of theophylline on ventilator-induced diaphragmatic dysfunction. J Crit Care. 2016 Jun;33:145-50. doi: 10.1016/j.jcrc.2016.01.007.

18. Jung B, Moury PH, Mahul M, de Jong A, Galia F, Prades A, Albaladejo $P$, Chanques $G$, Molinari N, Jaber S. Diaphragmatic dysfunction in patients with ICU-acquired weakness and its impact on extubation failure. Intensive Care Med. 2016 May;42(5):853861. doi: 10.1007/s00134-015-4125-2.

19. Dubé BP, Dres M, Mayaux J, Demiri S, Similowski T, Demoule A. Ultrasound evaluation of diaphragm function in mechanically ventilated patients: comparison to phrenic stimulation and prognostic implications. Thorax. 2017 Sep;72(9):811-818. doi: 10.1136/thoraxjnl-2016-209459.

20. Zambon M, Greco M, Bocchino S, Cabrini L, Beccaria PF, Zangrillo A. Assessment of diaphragmatic dysfunction in the critically ill patient with ultrasound: a systematic review. Intensive Care Med. 2017 Jan;43(1):29-38. doi: 10.1007/s00134-016-4524$z$.

21. Petrof BJ. Diaphragm Weakness in the Critically III: Basic Mechanisms Reveal Therapeutic Opportunities. Chest. 2018 Dec;154(6):1395-1403. doi: 10.1016/j.chest.2018.08.1028.

22. Levine S, Nguyen T, Taylor N, et al. Rapid Disuse Atrophy of Diaphragm Fibers in Mechanically Ventilated Humans. N Engl J Med. 2008;358(13):1327-1335.

23. Dres M, Dubé BP, Mayaux J, Delemazure J, Reuter D, Brochard $L$, Similowski $T$, Demoule A. Coexistence and Impact of Limb Muscle and Diaphragm Weakness at Time of Liberation from Mechanical Ventilation in Medical Intensive Care Unit Patients. Am J Respir Crit Care Med. 2017 Jan 1;195(1):57-66. doi: 10.1164/rccm.201602-0367OC.

24. Lu Z, Xu Q, Yuan Y, Zhang G, Guo F, Ge H. Diaphragmatic Dysfunction Is Characterized by Increased Duration of Mechanical Ventilation in Subjects With Prolonged Weaning. Respir Care. 2016 Oct;61(10):1316-22. doi: 10.4187/respcare.04746.

25. Mariani LF, Bedel J, Gros A, Lerolle N, Milojevic K, Laurent V, Hilly J, Troché G, Bedos JP, Planquette B. Ultrasonography for Screening and Follow-Up of Diaphragmatic Dysfunction in the ICU: A Pilot Study. J Intensive Care Med. 2016 Jun;31(5):338-43. doi: $10.1177 / 0885066615583639$.

26. Laghi F, Cattapan SE, Jubran A, Parthasarathy S, Warshawsky $P$, Choi YS, Tobin MJ. Is weaning failure caused by low-frequency fatigue of the diaphragm? Am J Respir Crit Care Med. 2003 Jan 15;167(2):120-7. doi: 10.1164/rccm.200210-1246OC.
27. Demoule A, Jung B, Prodanovic $H$, Molinari $N$, Chanques $G$, Coirault $C$, Matecki $S$, Duguet A, Similowski $T$, Jaber $S$. Diaphragm dysfunction on admission to the intensive care unit. Prevalence, risk factors, and prognostic impact-a prospective study. Am J Respir Crit Care Med. 2013 Jul 15;188(2):213-9. doi: 10.1164/rccm.201209-1668OC.

28. Demoule A, Molinari $N$, Jung B, Prodanovic $H$, Chanques $G$, Matecki S, Mayaux J, Similowski T, Jaber S. Patterns of diaphragm function in critically ill patients receiving prolonged mechanical ventilation: a prospective longitudinal study. Ann Intensive Care. 2016 Dec;6(1):75. doi: 10.1186/s13613-016-01798.

29. Goligher EC, Dres M, Fan E, Rubenfeld GD, Scales DC, Herridge MS, Vorona S, Sklar MC, Rittayamai N, Lanys A, Murray $A$, Brace $D$, Urrea $C$, Reid WD, Tomlinson G, Slutsky AS, Kavanagh BP, Brochard LJ, Ferguson ND. Mechanical Ventilationinduced Diaphragm Atrophy Strongly Impacts Clinical Outcomes. Am J Respir Crit Care Med. 2018 Jan 15;197(2):204-213. doi: 10.1164/rccm.201703-0536OC.

30. Watson AC, Hughes PD, Louise Harris M, Hart N, Ware RJ, Wendon J, Green M, Moxham J. Measurement of twitch transdiaphragmatic, esophageal, and endotracheal tube pressure with bilateral anterolateral magnetic phrenic nerve stimulation in patients in the intensive care unit. Crit Care Med. 2001 Jul;29(7):1325-31. doi: 10.1097/00003246-200107000-00005.

31. Goligher EC, Laghi F, Detsky ME, Farias $P$, Murray A, Brace $D$, Brochard LJ, Bolz SS, Rubenfeld GD, Kavanagh BP, Ferguson $N D$. Measuring diaphragm thickness with ultrasound in mechanically ventilated patients: feasibility, reproducibility and validity. Intensive Care Med. 2015 Apr;41(4):642-9. doi: 10.1007/s00134-015-3687-3. Erratum in: Intensive Care Med. 2015 Apr;41(4):734. Sebastien-Bolz, Steffen [corrected to Bolz, Steffen-Sebastien]

32. Hermans G, Agten A, Testelmans D, Decramer M, GayanRamirez G. Increased duration of mechanical ventilation is associated with decreased diaphragmatic force: a prospective observational study. Crit Care. 2010;14(4):R127. doi: 10.1186/cc9094.

33. American Thoracic Society/European Respiratory Society. ATS/ERS Statement on respiratory muscle testing. Am J Respir Crit Care Med. 2002 Aug 15;166(4):518-624. doi: 10.1164/rccm.166.4.518.

34. Matamis D, Soilemezi E, Tsagourias M, Akoumianaki E, Dimassi S, Boroli F, Richard JC, Brochard L. Sonographic evaluation of the diaphragm in critically ill patients. Technique and clinical applications. Intensive Care Med. 2013 May;39(5):801-10. doi: 10.1007/s00134-013-2823-1.

35. Kim WY, Lim CM. Ventilator-Induced Diaphragmatic Dysfunction: Diagnosis and Role of Pharmacological Agents. Respir Care. 2017 Nov;62(11):1485-1491. doi: 10.4187/respcare.05622.

36. Baldwin CE, Paratz JD, Bersten AD. Diaphragm and peripheral muscle thickness on ultrasound: intra-rater reliability and variability of a methodology using non-standard recumbent positions. Respirology. 2011 Oct;16(7):1136-43. doi: 10.1111/j.14401843.2011.02005.x. 